\title{
Review of Polynomial Chaos-Based Methods for Uncertainty Quantification in Modern Integrated Circuits
}

\author{
Arun Kaintura *, Tom Dhaene (1) and Domenico Spina \\ IDLab, Department of Information Technology (INTEC), Ghent University-imec, \\ Technologiepark-Zwijnaarde 15, 9052 Ghent, Belgium; tom.dhaene@ugent.be (T.D.); \\ domenico.spina@ugent.be (D.S.) \\ * Correspondence: arun.kaintura@ugent.be; Tel.: +32-489763265
}

Received: 16 February 2018; Accepted: 26 February 2018; Published: 28 February 2018

\begin{abstract}
Advances in manufacturing process technology are key ensembles for the production of integrated circuits in the sub-micrometer region. It is of paramount importance to assess the effects of tolerances in the manufacturing process on the performance of modern integrated circuits. The polynomial chaos expansion has emerged as a suitable alternative to standard Monte Carlo-based methods that are accurate, but computationally cumbersome. This paper provides an overview of the most recent developments and challenges in the application of polynomial chaos-based techniques for uncertainty quantification in integrated circuits, with particular focus on high-dimensional problems.
\end{abstract}

Keywords: polynomial chaos; uncertainty quantification; integrated circuits; high dimensionality

\section{Introduction}

Nowadays, micro-electronic circuits are widespread and are used in many activities for personal and professional use: communication (smartphones), entertainment (personal computer), and spatial exploration (satellites) are just a few examples. The rapid development of modern high-speed integrated circuits (ICs) is supported by the technological innovations allowing integration of several sub-devices (such as digital, analog, micro electro-mechanical systems (MEMS), and radio frequency (RF) devices) on a single chip. Starting in the early nineties, the steady down-scaling of the device dimensions along with the integration of more components on a single chip have been the major sources of growth for modern micro-electronic circuits. This growth is in adherence to the Gordon Moore prediction, also known as Moore's Law, stating that the available memory and calculation speed of microprocessors would have an exponential growth doubling every year [1], and it still stands with the correction of doubling every 18 months [2].

These miniaturized ICs are not only cost-efficient but also offer high computational power, functionality, and portability coupled with low power dissipation, driving progress in the microelectronics industry to higher scaling of electronic circuits.

With the progress in the integration level of ICs moving towards a hundred million transistors in the next few years, the complexity of such circuits will also increase. Furthermore, such progress also resulted in an exponentially growing cost of designing, testing, and manufacturing equipment [3]. Mass production and efficient design strategies are the principal methods considered to reduce production costs and to introduce ICs in the market at a competitive price. In particular, the advances in manufacturing process technology is one of the core factors that make manufacturing ICs in the sub-micrometer region possible. However, various technological challenges emerge when such small geometries are used to realize large-scale ICs [4]. 
Hence, the robust design of modern ICs is fundamental, especially considering that the impact on the circuit performance in terms of geometrical or electrical parameter variations due to uncertainty sources has become increasingly relevant in the nanometer region [5-7]. Different types of variations are present, such as routing/layout uncertainties, and changes in a device's operating conditions due to temperature fluctuations; however, the tolerances of the manufacturing process rank among the major ones. Fundamentally, during the manufacturing process, a mismatch is introduced between devices that are designed to be the same. Moreover, different manufacturing phases are affected by process variations, such as etching, lithography, and polishing [8]. Hence, these uncertainties have become a major concern in IC design [6,9-11], and the process of identifying the effect on the circuit performance of geometrical or electrical parameters variability is generally referred to as uncertainty quantification (UQ) or uncertainty analysis.

Typically, Monte Carlo (MC)-based methods [12] are used as a standard approach to UQ thanks to their accuracy and ease of implementation. Basically, such approaches are sampling-based techniques: first, the circuit under study is simulated via suitable computer-aided design (CAD) programs, depending on the type of system under study (for example, electromagnetic simulators, such as ADS Momentum (Momentum EEsof EDA, Agilent Technologies, Santa Rosa, CA, USA), or circuit simulators, such as PSpice (Cadence Design Systems, San Jose, CA, USA) circuit simulators can be adopted), for a large set of samples of the random parameters considered, chosen according to their pertinent distributions. Then, based on the data obtained so far, the statistics and uncertainty analysis can be computed. Unfortunately, MC simulations require a substantial number of samples to converge, since it can be proved that the accuracy of the UQ results performed via the MC method depends on the inverse square root of the number of simulations performed. Since CAD simulations are often computationally expensive, due to the increased complexity of modern ICs, the MC method can be computationally prohibitive.

Alternatively, there has been an increasing interest in applying stochastic spectral methods as an efficient alternative to the computationally cumbersome MC-based techniques for UQ in circuit simulations: such as the polynomial chaos (PC) expansion $[13,14]$, stochastic collocation (SC) methods $[15,16]$, and stochastic reduced order models (SROMs) $[17,18]$. Among the different techniques presented in the literature, PC-based methods have attracted great interest in the research community for three main reasons: the capability to tackle UQ problems defined in the frequencyand time-domain considering different types of systems, linear and nonlinear, and involving random variables of different distributions, even in the presence of correlation; the efficiency in estimating stochastic moments (i.e., the mean and standard deviation can be computed via suitable analytical formulas); and the ease of integration in modern CAD tools, for example, via non-intrusive approaches or via the calculation of suitable equivalent circuits describing the variability of the system under study. All these characteristics will be described below. Thanks to their accuracy, efficiency, modeling power, and flexibility, the number of publications and citations on PC-based techniques for applications in the electrical and electronics fields has been sharply increasing over the last years, see Figure 1.

The objective of this paper is to provide an overview of the most recent developments in the application of the PC expansion for the UQ of modern ICs. In particular, this study does not focus on giving a complete description of the mathematical properties of the PC expansion, which have been thoroughly discussed in the literature, see, for example, [13,14,19-24]; rather, the goal is to provide the reader with an illustration of the reasons behind the success of PC-based approaches and a description of the unique features of the application of the such methods for different electromagnetic and electronic UQ applications. This work is organized as follows: the characteristics of process-induced variations are presented in Section 2, while an overview of the PC expansion method is given in Section 3, along with a description of the challenges associated with a PC model construction. Section 4 highlights recent developments and applications of PC-based methods of circuit simulations. Challenges and recent developments in high-dimensional applications are discussed in Section 5, while conclusions are summed up in Section 6. 


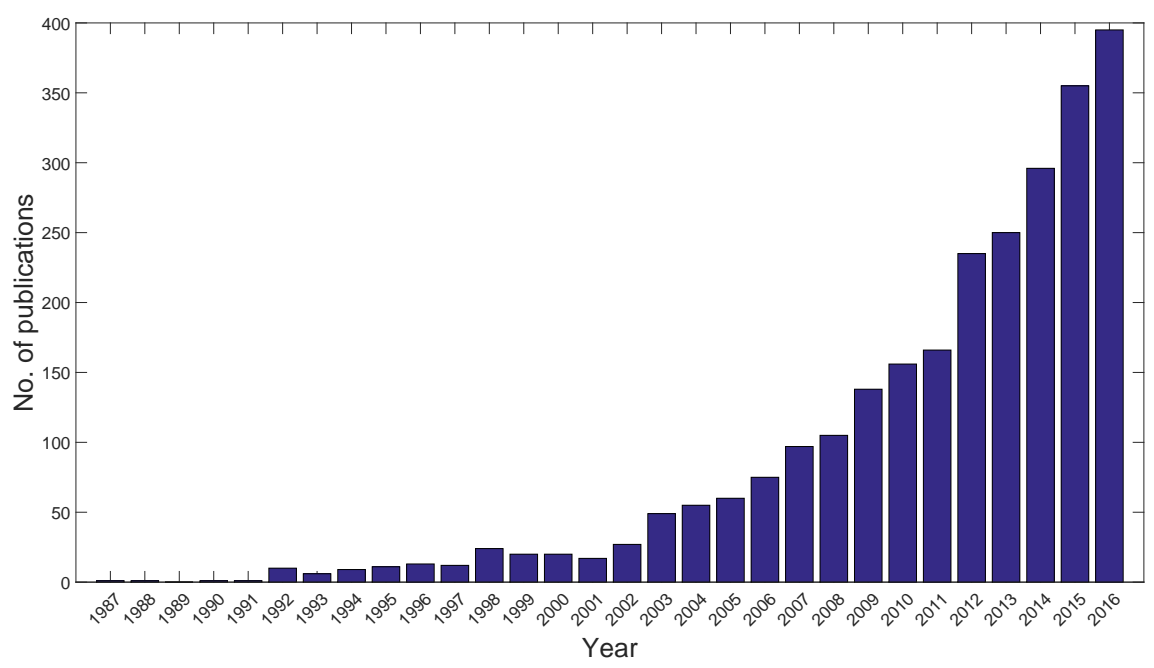

(a) Number of publications.

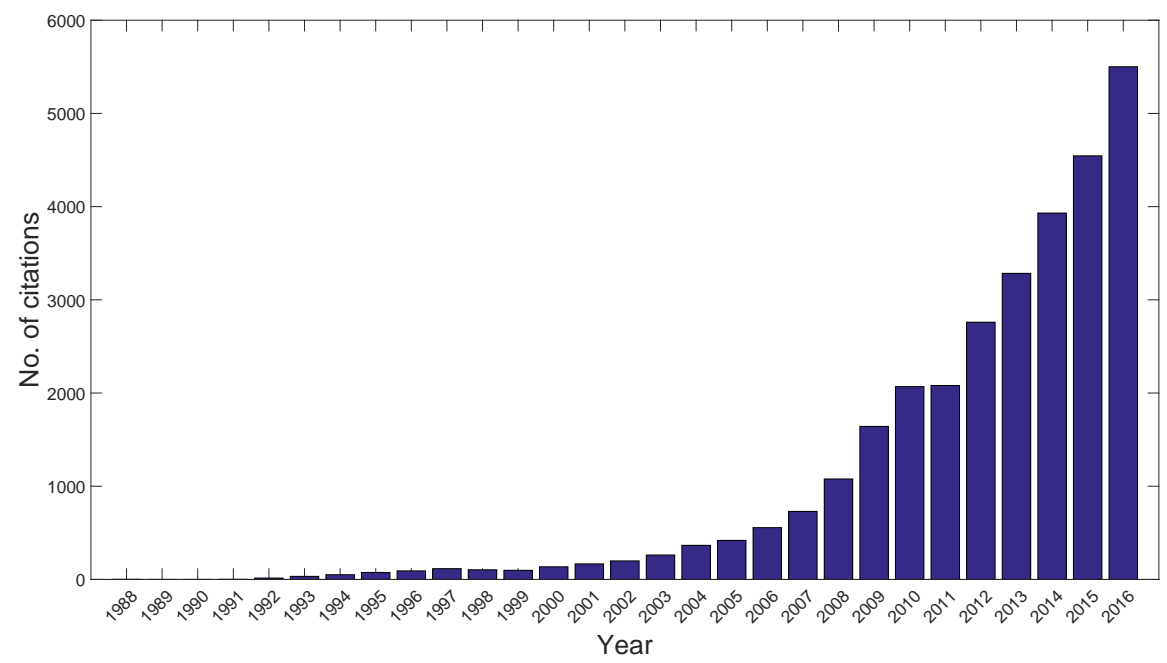

(b) Number of citations.

Figure 1. (a) Number of publications per year on PC-based methods for applications in the electrical and electronic domains, indexed in the ISI Web of Science [25]. (b) Total number of citations of PC-based methods for applications in the electrical and electronic domains, indexed in the ISI Web of Science [25]

\section{Problem Setting}

In general, two main types of uncertainty sources affect the behavior of modern ICs: temporal and spatial [26]. The first are time-dependent effects, which can be divided into wearout or aging, which permanently degrade the circuit performance over time (e.g., hot carrier injection), and transient disturbances, which manifest only for a limited period of time and, usually, do not permanently deteriorate the circuit performance (such as electromagnetic interference). Spatial variability, instead, is caused by the fabrication process of modern ICs: its effects manifest immediately after production and are fixed over time. In particular, spatial uncertainty effects can be divided into two categories: the ones causing a mismatch (intradie variations) between devices on the same die that are designed to be identical, and the interdie ones causing variations between devices separated by a long distance (from die to die) or that are manufactured at a different time (between wafer to wafer, or lot to lot) [26]. Typically, interdie variations cause a shift in the mean value of the chosen design parameters [26], while 
process-induced mismatch significantly threatens the efficiency of digital circuits, by influencing their timing closure [8], and analog ones, since matched devices and differential signal paths are fundamental for most high-performance analog circuits [27]. Hence, this contribution focuses on analyzing the mismatch resulting from the tolerances of the manufacturing process.

In this scenario, excluding systematic or epistemic uncertainty sources (which are uncertainties due to a lack of knowledge), the device-related sources of spatial unreliability can be described as random [26] and can be characterized in a statistical way. Hence, the process-induced variations can be represented by considering the geometrical (i.e., the width and length of an interconnection and the thickness of a dielectric material) or electrical (i.e., the threshold voltage of a transistor and the relative permittivity of the substrate) parameters of the device under study as random variables with a suitable distribution. The nominal value of these random variables represents the value chosen during the design phase, while the probability distribution describes the likelihood for a chosen parameter to assume specific value close to its nominal one. In this framework, the performance of the circuit under study is no longer deterministic anymore and can be characterized via suitable UQ techniques, see Figure 2.

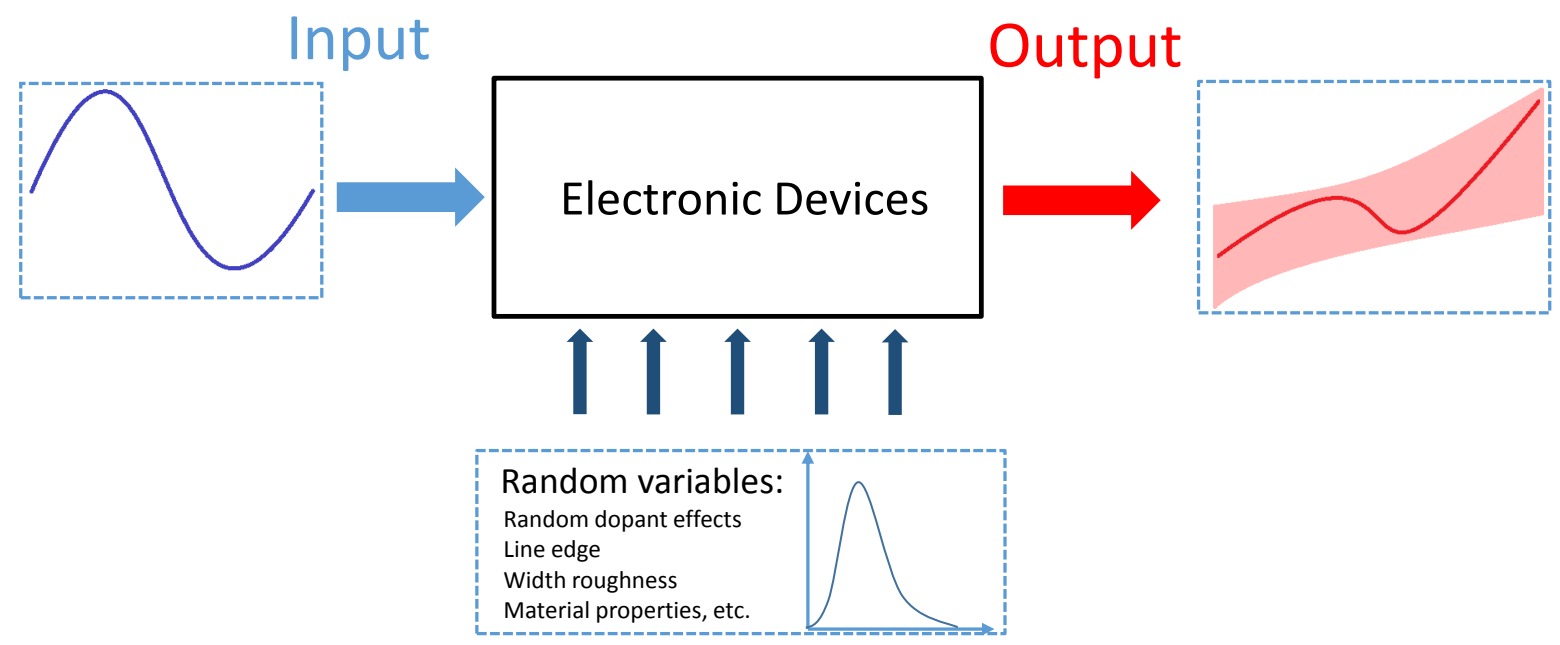

Figure 2. Example of the effect of uncertain parameters on a system response. In the presence of random variables, the response to a deterministic stimulus is not deterministic anymore and must be characterized in a statistical manner: the red line denotes the nominal response, while the gray area indicates its fluctuation.

Hence, the main goal of UQ is to quantify the effect of the process variations on the circuit performances, which can be described in terms of the port's voltage and current, and other specific performance indicators of the device under study, such as the gain and total harmonic distortion of an RF amplifier or the quality factor of an inductor. To this aim, the PC expansion builds a mathematical model of the stochastic variations in the circuit considered, starting only from the knowledge of the distribution of the random parameters considered, which is able to accurately and efficiently describe the stochastic variations in the circuit performance.

\section{Polynomial Chaos Expansion}

In 1938, Wiener [19] discussed the role of Hermite polynomials and homogeneous chaos in the integration theory with respect to the Brownian motion. However, the research based on the PC expansion received a new impulse in the last twenty years, see Figure 1, thanks to recent research developments. For example, in [20], the Hermite-chaos has been applied in combination with the finite element method to address uncertainties in various problems in solid mechanics, one of the first successful applications in the early nineties, while in [13] an optimal Askey scheme-based PC (or generalized polynomial chaos ( $\mathrm{gPC}$ )) has been proposed to study stochastic processes depending 
on random variables with different distributions. The theory of the PC expansion has strong mathematical rigor and complexity, which has been studied in several publications in the literature, see, for example, [13,14,19-24,28]. However, considering the aim of this review paper, only an overview of the most relevant properties of the PC expansion will be given in the following, in order to focus on its application for UQ problems in the electrical and electronics domains. Suitable references are provided.

\subsection{Preliminaries: PC Properties}

The PC expansion can be considered as a mathematical model that is able to solve stochastic problems: the response of a system under stochastic effects can be represented via some properly constructed basis functions.

Indeed, let us consider a stochastic process $\mathrm{Y}(x, t, \xi)$ with finite second-order moments, depending on the normalized random variables collected in the vector $\xi$ :

$$
Y(x, t, \xi)=\mathscr{L}(x, t, \xi)
$$

where $t \in[0, T]$ represents the time, $x$ is the state vector, and $\mathscr{L}$ is an operator (linear or nonlinear). Now, $Y$ can be expressed as an infinite series of orthogonal basis functions $\varphi_{i}$ with suitable coefficients $\alpha_{i}$ as $[13,14,23]$

$$
\mathrm{Y}(t, \xi)=\sum_{i=0}^{\infty} \alpha_{i}(t) \varphi_{i}(\xi)
$$

where the representation in (2) is exact. However, for practical applications, Equation (2) must be truncated to a limited number of basis functions $\boldsymbol{M}+\mathbf{1}$ via suitable truncation schemes, as will be discussed in detail in the next sections, leading to

$$
\mathrm{Y}(t, \xi) \approx \sum_{i=0}^{M} \alpha_{i}(t) \varphi_{i}(\xi)
$$

Furthermore, the basis functions follow the orthogonality conditions:

$$
<\varphi_{i}(\xi), \varphi_{j}(\xi)>=\int_{\Omega} \varphi_{i}(\xi) \varphi_{j}(\xi) W(\xi) \mathrm{d} \xi=a_{i} \delta_{i j} \quad \text { for } i, j=0, \ldots, M
$$

where $\left\langle\cdot, \cdot>\right.$ denotes the inner product, $a_{i}$ are real numbers, $\delta_{i j}$ is the Kronecker Delta function, and $W(\xi)$, the weighting function in the theory of orthogonal polynomials [13,23], is a probability measure with support $\boldsymbol{\Omega}$. Note that Equation (4) defines an inner product in the stochastic space $\boldsymbol{\Omega}$, which is the domain of variation in the random variables $\xi$ : when more than one random variable is considered, such inner product is a multi-dimensional integral. Furthermore, a set of orthonormal basis functions can always be obtained via standard normalization procedures: in this case, $\boldsymbol{a}_{i}=\mathbf{1}$ for $i, j=0, \ldots, \boldsymbol{M}$. Without loss of generality, the basis functions $\varphi_{i}$ will be considered as an orthonormal set in the rest of the publication.

When applying the PC expansion for the UQ of electronic circuits, the stochastic process $Y$ represents the quantities of interest (the voltage and the current at the input and output ports of the circuit, for example), while the geometrical or electrical parameters varying due to the tolerances of the manufacturing process are described by the random variables $\xi$ (for example, the threshold voltage or the channel width of the circuit transistors). It is important to note that Equation (3) can also be formulated in the frequency domain, by simply replacing the time $t$ with the frequency $f$ : in this case, the PC expansion can also be applied to analyze the variations in the frequency response (described by different representations, such as scattering, impedance, or admittance parameters) of any passive multiport circuits (such as interconnections, distributed filters, and connectors).

One of the main reasons behind the success of PC-based methods is the ability to perform UQ of complex circuits with high accuracy and efficiency. Indeed, once a PC model in the form of Equation (3) 
is obtained, stochastic moments like the mean $\mu$ and variance $\sigma^{2}$ of $Y$ can be analytically calculated based on the PC expansion coefficients as [23]

$$
\begin{gathered}
\mu(\mathrm{Y}(t, \xi))=\alpha_{0}(t) \\
\sigma^{2}(\mathrm{Y}(t, \xi))=\sum_{i=1}^{M} \alpha_{i}^{2}(t) .
\end{gathered}
$$

Apart from all moments, complex stochastic functions of $\boldsymbol{Y}$, such as the cumulative distribution function (CDF) and probability density function (PDF), can be efficiently obtained via the (inexpensive) sampling of (3). Moreover, the PC model can also be used for sensitivity analysis, and PC-based SOBOL indices can be determined easily from the PC expansion coefficients [29-31].

\subsection{Computing a PC Model}

The first step to obtain a model in the form of Equation (3) is to estimate the basis functions $\varphi_{i}$, which are typically polynomial functions of $\xi$, which will be described in the following. A fundamental property of the PC method is that the basis functions must satisfy the orthogonality relation (4). In general, the inner product in (4) can be defined for different types of weighting function $W(\xi)$; however, it is possible to prove that the optimal convergence rate of a PC model can be achieved when the weighting function $W(\xi)$ corresponds to the joint PDF of the random variables considered expressed in a standard form, since the random variables $\xi$ are normalized [13,32]. In this framework, an optimal convergence rate means that the minimum number of basis functions $\boldsymbol{M}+\mathbf{1}$ is necessary to obtain an accurate PC model (3). Examples of weighting functions for different types of random variables are given in Table 1.

Table 1. Polynomials of the Wiener-Askey scheme for some random variables $[13,33]$. Note that $\Gamma$ and $\beta$ are the Gamma and Beta functions, respectively.

\begin{tabular}{ccccc}
\hline Distribution & Orthogonal Polynomials & PDF & Weight Function & Support Range \\
\hline Gaussian & Hermite $H_{e_{k}}(\xi)$ & $\frac{1}{\sqrt{2 \pi}} e^{\frac{-x^{2}}{2}}$ & $e^{\frac{-x^{2}}{2}}$ & {$[-\infty, \infty]$} \\
Uniform & Legendre $P_{k}(\xi)$ & $\frac{1}{2}$ & 1 & {$[-1,1]$} \\
Gamma & Laguerre $L_{k}^{a}(\xi)$ & $\frac{x^{a} e^{-x}}{\Gamma(a+1)}$ & $x^{a} e^{-x}$ & {$[0, \infty]$} \\
Beta & Jacobi $J_{k}^{a, b}(\xi)$ & $\frac{(1-x)^{a}(1+x)^{b}}{2^{a+b+1} \beta(a+1, b+1)}$ & $(1-x)^{a}(1+x)^{b}$ & {$[-1,1]$} \\
\hline
\end{tabular}

Hence, the choice of the basis functions depends only on the distribution of the random variables $\xi$ (more specifically on their joint PDF), and it is not influenced by the type of system under study (i.e., mixer, oscillator, multistage amplifier, microwave filter, etc.). In particular, if the random variables $\xi$ are independent, their joint PDF corresponds to the product of the distributions of each random variable: in this case, the corresponding basis functions $\varphi_{i}$ can be calculated as product combinations of the orthogonal polynomials corresponding to each individual random variable $\phi$ [23]:

$$
\varphi_{j}(\xi)=\prod_{k=1}^{N} \phi_{i_{k}}\left(\xi_{k}\right) \quad \sum_{k=1}^{N} i_{k} \leq P \quad \text { and } \quad 0 \leq j \leq M
$$

where $\boldsymbol{N}$ is the number of random variables considered, and $\boldsymbol{P}$ is the maximum order of the polynomials in (3). Furthermore, for random variables with specific PDFs (indicated in the sequel as standard distributions), the optimal basis functions are known and are formed by the polynomials of the Wiener-Askey scheme [13], as shown in Table 1. For example, in the Gaussian PDF case, the basis 
functions are the Hermite polynomials; in the uniform PDF case, the basis functions are the Legendre polynomials.

Now, since the PC basis functions are polynomials, they also include a constant term (corresponding to a polynomial of degree zero). Such basis function is usually indicated as $\varphi_{0}(\xi)$, and it is possible to prove that $\varphi_{0}(\xi)=\mathbf{1}$ for orthonormal basis functions. Indeed, Equation (4) for $i, j=\mathbf{0}$ becomes

$$
<\varphi_{0}(\xi), \varphi_{0}(\xi)>=\int_{\Omega} \varphi_{0}(\xi) \varphi_{0}(\xi) W(\xi) \mathrm{d} \xi
$$

Let us assume $\varphi_{0}(\xi)=c$, where $c$ is an arbitrary real number. Hence, Equation (8) can be written as

$$
<\varphi_{0}(\xi), \varphi_{0}(\xi)>=c^{2} \int_{\Omega} W(\xi) \mathrm{d} \xi
$$

Since $W(\xi)$ is a probability density function (as discussed above), the integral of such a function over the entire stochastic space is equal to 1 , by definition. Hence, it is possible to write

$$
<\varphi_{0}(\xi), \varphi_{0}(\xi)>=c^{2}
$$

It follows that $c=\mathbf{1}$ for orthonormal basis functions.

At this point, it is easy to derive Equations (5) and (6). Indeed, Equation (5) is given by

$$
\mu(\mathrm{Y}(t, \xi))=\int_{\Omega} \mathrm{Y}(t, \xi) W(\xi) \mathrm{d} \xi .
$$

Since $\varphi_{0}(\xi)=1$, it is possible to write

$$
\mu(\mathrm{Y}(t, \xi))=\int_{\Omega} \mathrm{Y}(t, \xi) \varphi_{0}(\xi) W(\xi) \mathrm{d} \xi=<\mathrm{Y}(t, \xi), \varphi_{0}(\xi)>
$$

By expressing $\mathrm{Y}(t, \xi)$ via its PC model (3) and by considering the orthogonality of the PC basis functions (4), leads to

$$
\mu(\mathrm{Y}(t, \xi))=<\mathrm{Y}(t, \xi), \varphi_{0}(\xi)>=\alpha_{0}(t) .
$$

Similarly, Equation (6) can be derived as

$$
\begin{aligned}
\sigma^{2}(\mathrm{Y}(t, \xi)) & =\int_{\Omega}[\mathrm{Y}(t, \xi)-\mu(\mathrm{Y}(t, \xi))]^{2} W(\xi) \mathrm{d} \xi \\
& =\int_{\Omega}\left[\mathrm{Y}^{2}(t, \xi)+\mu^{2}(\mathrm{Y}(t, \xi))-2 \mathrm{Y}(t, \xi) \mu(\mathrm{Y}(t, \xi))\right] W(\xi) \mathrm{d} \xi \\
& =\int_{\Omega}\left[\mathrm{Y}^{2}(t, \xi)+\alpha_{0}^{2}(t)-2 \alpha_{0}(t) \mathrm{Y}(t, \xi)\right] W(\xi) \mathrm{d} \xi \\
& =<\mathrm{Y}(t, \xi), \mathrm{Y}(t, \xi)>+\alpha_{0}^{2}(t)-2 \alpha_{0}^{2}(t) .
\end{aligned}
$$

Thanks to Equation (4), it is possible to write

$$
\sigma^{2}(\mathrm{Y}(t, \xi))=\sum_{i=0}^{M} \alpha_{i}^{2}(t)-\alpha_{0}^{2}(t)=\sum_{i=1}^{M} \alpha_{i}^{2}(t) .
$$

Now, for random variables with arbitrary distribution, the basis functions can be computed via several techniques, such as $[32,34,35]$. It is important to note that PC models can also be obtained if the random variables $\xi$ are correlated $[13,23]$ : indeed, approaches based on suitable variable transformations (such as the Karhunen-Loéve expansion [36] or the copula theory [14]) can be adopted. Hence, without loss of generality, in the rest of the section only the case of independent random variables will be considered. 
Once the basis functions have been estimated, the next step is to truncate the infinite PC expansion (2) to a limited number of $\boldsymbol{M}+\mathbf{1}$ terms. Since the independent random variable Equation (7) holds, it is possible to prove that the total number of basis functions $\boldsymbol{M}+\mathbf{1}$ in (3) can be expressed as

$$
M+1=\frac{(N+P) !}{N ! P !} .
$$

A PC model obtained via Equations (7) and (15) is formed by polynomials up to a maximum degree $\boldsymbol{P}$ (also called order of the PC model): this truncation strategy is usually referred to as a "total order expansion" [23]. Now the question arises as to how an order $\boldsymbol{P}$ that will give accurate results for the specific problem at hand can be chosen. It has been proven in the literature that a relatively small order $\mathbf{2}<\boldsymbol{P}<\mathbf{5}$ is sufficient to achieve the desired accuracy for many applications in the electronics domain, since the variations in the parameter values induced by the tolerance of the manufacturing process are relatively limited $[37,38]$. Hence, a popular strategy is to choose a priori the order of the expansion $\boldsymbol{P}$ for the problem at hand. Alternatively, an adaptive strategy can be adopted based on cross-validation in the estimation of stochastic moments, such as [39], or methods computing sparse PC models, where only the significant terms out of all the $\boldsymbol{M}+\mathbf{1}$ elements in (3) are used, see, for example, [40,41].

A different truncation approach is called "tensor-product expansion" [23]: the PC basis functions are formed by the product combination of the orthogonal polynomials corresponding to each individual random variable $\phi$, as in (7), but the maximum order of $\phi$ is chosen, rather than the maximum order of the overall basis functions $\varphi$, leading to

$$
M+1=\prod_{k=1}^{N}\left(p_{k}+1\right)
$$

where $p_{k}$ is the maximum degree of the polynomials corresponding to the $k$-th random variable. Note that the PC basis functions obtained via the "total order expansion" are a subset of the ones given by the "tensor-product expansion" by choosing $p_{k}=p$ for $k=1, \ldots, N$ and $p=P$. Given that one of the main limits of the PC expansion is the rapid increase of the terms in (3) with respect to the number of random variables and the order of the polynomial considered, as discussed in details in Section 5, the "total order expansion" is commonly adopted as the truncation strategy.

Finally, in order to obtain a PC model in the form of Equation (3), the coefficients $\alpha_{i}$ must be computed. Note that an accurate estimation of such coefficients is fundamental for UQ purposes: for example, stochastic moments such as mean and variance depend only on $\boldsymbol{\alpha}_{i}$, see Equations (5) and (6). However, differently from the basis functions, the PC coefficient estimation depends on the type of system considered.

Indeed, modern high-speed ICs rely on heterogeneous devices and components (i.e., connectors, operational amplifiers, filters, interconnections, etc.), where each type of electric and electronic component has its unique characteristics and corresponding set of specifications that designers must satisfy. Hence, it is possible to classify the different types of devices in broad classes, such as lumped or distributed, passive or active, and linear or nonlinear. Note that any electric and electronic device or component falls into multiple categories: a microstrip line is a distributed, linear, and passive element, while a typical operational amplifier is a lumped, nonlinear, and active device.

These distinctions reflect important physical differences in the device's behavior, leading to different methods used to characterize them: for example, general linear and passive systems are typically analyzed in the frequency domain by means of their transfer function, which can be expressed in different representations (i.e., impedance, admittance, or scattering parameters), while nonlinear (typically) active elements are mainly studied in the time domain. These differences require the adoption of suitable methodologies to characterize the specific device or component considered: the transfer function estimation is, in general, performed via full-wave electromagnetic simulations, while SPICE-like circuit simulators are adopted for the time-domain analyses. 
Hence, different PC-based techniques have been developed over the years by the research community tailored on the types of devices or components considered: lumped element circuits [42-44], multiconductor transmission lines [45-47], generic linear multiport systems [37,39,48], and nonlinear systems [38,49-53]. Additional details are given in the next section, where a review of the main applications of the PC method in electrical and electronics problem is presented.

\section{PC-Based Applications in Electronics}

In general, there are two possible strategies to compute the PC coefficients for a specific application at hand: intrusive or nonintrusive approaches. The first are sampling-based methods requiring to solve the problem at hand multiple times for specific values of the random variables considered, chosen according to a suitable sampling strategy [20,24]. Note that a deterministic problem is solved at each iteration. In this regard, this strategy is similar to MC-based methods, with the important difference that the number of samples required for the computation of PC models is limited (unless the number of random parameters considered is high, as it will be discussed in Section 5). The latter requires modification of the fundamental equations governing the system under study (e.g., Telegrapher's or modified nodal equations) in order to obtain a new system of equations where the desired PC coefficients are the unknowns [23]. The main difference between these two approaches is that nonintrusive techniques are easy to implement and use existing code as black-box (no modifications of the system fundamental equations are required), while intrusive ones generally lead to a coupled system of equations, which can be more expensive to solve with respect to the original problem, but all the PC coefficients can be determined in a single run.

In the following, an overview of the characteristics of some popular PC-based techniques is given, including spectral projection [13,23], linear regression [23,54,55], Galerkin projection-based approaches [24,56,57], and stochastic testing [38,58,59].

- Spectral Projection or Collocation Approach: By definition, the PC coefficients $\alpha_{i}$ can be expressed as $[13,23]$

$$
\alpha_{i}(t)=\left\langle\mathrm{Y}(t, \xi), \varphi_{i}(\xi)\right\rangle \quad \text { for } i=0, \ldots, M
$$

Note that Equation (17) can be directly obtained by projecting Equation (3) onto each basis function $\varphi_{i}$ for $i=0, \ldots, M$ and by employing the orthonormal relation of Equation (4) (where $a_{i}=\mathbf{1}$ in Equation (4) for orthonormal basis functions). Now, the inner product in Equation (17) can be written as a suitable multi-dimensional integral (see Equation (4)):

$$
\alpha_{i}(t)=\int_{\Omega} \mathrm{Y}(t, \xi) \varphi_{i}(\xi) W(\xi) \mathrm{d} \xi \quad \text { for } i=0, \ldots, M .
$$

In general, it is not possible to solve Equation (18) analytically, but numerical integration techniques can be adopted. Indeed, let us consider a set of $K$ values of the random variables $\xi$, indicated with $\xi_{k}$ for $k=1, \ldots, K$, and the corresponding deterministic solutions $\mathrm{Y}\left(t, \xi_{k}\right)$; Equation (18) can then be expressed as

$$
\alpha_{i}(t)=\int_{\Omega} \mathrm{Y}(t, \xi) \varphi_{i}(\xi) W(\xi) \mathrm{d} \xi \approx \sum_{k=1}^{K} \mathrm{Y}\left(t, \xi_{k}\right) \varphi_{i}\left(\xi_{k}\right) w_{k} \quad \text { for } i=0, \ldots, M
$$

where the values $\xi_{k}$ for $k=1, \ldots, K$ (also called quadrature points) and corresponding weights $w_{k}$ depend on the particular numerical method adopted [60]. For example, the tensor-product approach is an efficient technique when the number of random variables is small, whereas sparse grid techniques (e.g., the Smolyak algorithm [61]) are proved to be more efficient for a large number of random variables (see Appendix A). Note that various adaptive methods for quadrature node selection have also been introduced in the literature: for example, in [62], an adaptive algorithm based on nested sparse grids is proposed and applied to a stochastic 
eddy current NDT problems, a weighted Smolyak algorithm is presented in [63], an adaptive hierarchical sparse grid collocation algorithm and a data-driven sparse grid approach are illustrated in [64,65], respectively. In conclusion, a spectral projection can be considered as a nonintrusive strategy, requiring a suitable deterministic problem to be solved $\boldsymbol{K}$ times $[23,66]$.

- Linear regression is a nonintrusive approach whereby the PC coefficients are computed via a suitable least square problem [23,54,55]. Indeed, let us consider again a set of $\boldsymbol{K}$ samples of our random variables $\xi_{k}$ for $k=1, \ldots, K$, the corresponding values of the basis functions $\varphi_{i}\left(\xi_{k}\right)$ and of the stochastic process $\mathrm{Y}\left(\boldsymbol{t}, \boldsymbol{\xi}_{\boldsymbol{k}}\right)$. Now, by looking at Equation (3), a suitable linear system can be written as

$$
\vec{\varphi}\left(\xi_{k}\right) \vec{\alpha}(t)=\overrightarrow{\mathrm{Y}}\left(t, \xi_{k}\right)
$$

where $\vec{\varphi}\left(\xi_{k}\right)$ and $\vec{Y}\left(t, \xi_{k}\right)$ contain the basis functions $\varphi_{i}\left(\xi_{k}\right)$ and realizations $\mathbf{Y}\left(t, \xi_{k}\right)$ computed for the chosen $K$ values of the random variables, respectively, and $\vec{\alpha}(t)$ collects the PC coefficients to be determined.

If the number of $K$ samples is greater than the $M+1$ unknown PC coefficients, then the system (20) is over-determined and can be solved as a least square problem [54]. Generally, a value $K \approx 2(M+1)$ is considered to be sufficient for a robust solution and to prevent overfitting [67]. Linear regression has been a popular technique to determine the PC coefficients in various stochastic problems in circuit analysis. However, the choice of the regression nodes highly impact the accuracy of the results. As a result, various criteria have been proposed in the literature, such as random sampling and Latin Hypercube-based approaches [68], which suffer from slow convergence. An efficient D-optimal approach based on the optimization of a suitable information matrix has been proposed [69]. Moreover, a sparse linear regression method for high-speed circuits based on the modified Fedorov search algorithm is described in [70], which utilizes comparatively few regression nodes for the PC coefficient computation. Linear regression has been used with optimal regression nodes based on D-optimal design for microwave/RF networks in a multi-dimensional UQ framework [71]. In [72], the regression nodes are selected by a non-adaptive quasi-optimal technique (for least squares linear regression), which maximizes a parameter based on the mutual column orthogonality and the determinant of the model matrix. Various coherence-based random sampling schemes has also been derived from the properties of Hermite and Legendre polynomials as proposed in [73].

- The Galerkin Projection method is an intrusive method for evaluating the desired PC coefficients. As already mentioned, it generates a coupled system of deterministic equations where the PC coefficients are the unknowns, and these equations can be solved by using suitable numerical methods. Indeed, let us consider a general system of equations in the form of Equation (1). Expressing the quantities in Equation (1) depending on the random variables $\xi$ by means of the PC expansion leads to

$$
Y(x, t, \xi)=\mathscr{L}\left(x, t ; \sum_{i=0}^{M} \alpha_{i}(t) \varphi_{i}(\xi)\right) .
$$

Now, it is possible to project Equation (21) onto each PC basis function $\boldsymbol{\varphi}_{j}$ as

$$
\left\langle Y(x, t, \xi), \varphi_{j}(\xi)\right\rangle=\left\langle\mathscr{L}\left(x, t ; \sum_{i=0}^{M} \alpha_{i}(t) \varphi_{i}(\xi)\right), \varphi_{j}(\xi)\right\rangle \quad \text { for } j=0, \ldots, M .
$$

Note that starting from the initial stochastic problem of Equation (1), we have obtained a system of $\boldsymbol{M}+\mathbf{1}$ coupled deterministic equations in the form of Equation (22). Indeed, the projection operator $\langle\cdot, \cdot\rangle$ requires integration of the quantity of interest with respect to the random variables $\boldsymbol{\xi}$ in the stochastic space $\boldsymbol{\Omega}$, as shown in Equations (4) and (18). By solving such system of equations, the desired PC coefficients $\alpha_{i}$ can be determined. In particular, the obtained coupled 
system of equations has the same form of the original problem of Equation (1) when no stochastic effects are present: this problem can be solved by means of the same methods applicable to the "original" problem under study, depending on the type of (linear or nonlinear) operator $\mathscr{L}$ considered. Using the Galerkin Projection method offers an elegant way of computing the PC coefficients, allowing its application to a wide variety of problems. For example, such an intrusive approach has been adopted to solve transmission lines $[47,74]$ or stochastic full wave [75] problems. However, using the Galerkin method poses some challenges: Formulating and solving the obtained coupled system of equations for the determination of PC coefficients could be a difficult task. Furthermore, the method, for problems with a high-dimensional random space $[76,77]$ or when complex mathematical equations are considered, can result in a significant computational cost.

- Stochastic Testing in $[38,58]$, an efficient and accurate algorithm, was introduced for the UQ of transistor-level circuits, which has been implemented in a SPICE-type stochastic simulator. It utilizes a suitable set of $K$ values of the random variables $\xi$ to generate a new system of deterministic coupled equations as a function of the PC coefficients, starting from the initial stochastic equations. The first step is to select $K$ testing nodes $\xi_{k}$ for $k=1, \ldots, K$ (also known as collocation points) from a large pool of candidate nodes $(P+1)^{N}$ generated by a suitable collocation scheme (such as tensor product or sparse grid method [60], see the discussion in the collocation approach above). The most important nodes are then selected based on the testing node scheme in [38]. Hence, Equation (21) can be formulated as

$$
R(\vec{\alpha}(t), \xi)=\{Y(x, t, \xi)-\mathscr{L}(x, t ; Y)\}
$$

where $R$ is the residual function and $\vec{\alpha}(t)$ is the vector of the PC coefficients such that $\alpha_{k}(t)$ for $k=1, \ldots, K$. Finally, a system of coupled algebraic equations is generated by enforcing the residual function to be zero at each of the testing nodes. The resulting equation can then be directly solved by the stochastic testing solver in an intrusive way in order to estimate the PC coefficients.

One of the advantages of stochastic testing method over other approaches is its efficiency. Indeed, this method employs an efficient testing node scheme that significantly reduces the required number of samples in comparison to the traditional tensor-product and sparse grid-based methods [22,60]): $\boldsymbol{K}=\boldsymbol{M}+\mathbf{1}$. Moreover, a decoupling procedure applied inside the intrusive solver makes the stochastic testing method computationally more efficient than Galerkin-based approaches [38,58]. A nonintrusive formulation of the stochastic testing approach has been presented in [59], while in [78], the stochastic testing method is applied to evaluate the performance of a wireless links under the influence of a large number of random variables in a nonintrusive manner.

Note that PC-based methods have been extensively used for UQ problems of electrical and electronics circuits: many techniques have been developed addressing different types of problems. Giving a complete overview of all the techniques presented in the literature in the last fifteen years is prohibitive, given the high amount of publications in this area, see Figure 1. However, a brief list of significant applications is given in the following.

The multi-element generalized PC method (ME-gPC) was introduced to address discontinuities in the random space by decomposing the random inputs space into disjoint elements $[79,80]$. Multi-resolution schemes [81,82] allows one to represent the random variables in terms of polynomial multi-wavelets. In [83-85], the PC expansion has been combined with model order reduction techniques to study systems described by a large set of equations. In [86], stochastic spectral methods have been applied to a hierarchical UQ of integrated circuits, while a gradient enhanced PC for circuit simulation has been introduced in [87]. Recently, multi-fidelity UQ via PC has also received 
considerable interest [88]. In [89], a nonintrusive multi-fidelity approach, based on the regression method, has been presented.

Notably, several techniques are able to compute a deterministic PC-based equivalent circuit describing the performance variations in the original circuit under stochastic effects. In particular, the port voltages and currents of such equivalent circuits are the PC coefficients of the voltage and current at the port of the original circuit. Different techniques have been proposed, depending on the particular system under study (i.e., lumped elements and transmission lines [90], general linear and passive devices [48], and nonlinear circuits [59,91]). Such equivalent circuits are usually referred to as "augmented circuits" in the PC jargon, since their number of ports are higher with respect to the corresponding original circuit under stochastic effects.

\section{High-Dimensional Problems}

PC-based methods have been successfully adopted for different UQ problems with a relatively small number of random variables, see, for example, [13,22,24,38,47,48,70,74,92]. However, specific limitations and challenges arise in the application of the PC expansion when a high number of random variables is considered [93]. Note that the number of terms $\boldsymbol{M}+\mathbf{1}$ in a PC model increases rapidly with respect to the maximum degree of the polynomial basis functions $\boldsymbol{P}$ (also called order of the expansion) and the number of random parameter $N$, as shown in Equation (15). While the order of the expansion is usually limited for typical UQ problems due to the tolerances of the manufacturing process [37,38], as discussed in Section 3.2, the number of geometrical or electrical parameters under stochastic effects can be high, especially for complex circuits. Table 2 shows an example of the effect of the number of random parameters $N$ on the total number of terms in a PC model for different expansion orders: clearly, the PC model complexity rapidly grows with respect to $N$, even for a small degree of the polynomials (e.g., $\boldsymbol{P}=2$ ). Furthermore, the number of evaluations of the stochastic process under study $K$ to be performed in order to compute a PC model typically depends on $M+1$, as described in the previous section, for different intrusive and nonintrusive techniques. Since simulations (or measurements) of complex electromagnetic or electronic systems can be computational expensive, it is of paramount importance to keep $K$ as small as possible.

Hence, the number of random parameters has an effect on both the model complexity and computational cost of any PC-based methods, and, when $N$ is sufficiently high, the efficiency of such approaches can be greatly reduced. In this section, we discuss and highlight the most recent PC-related developments for UQ of high-dimensional problems by presenting techniques aiming at reducing the number of samples needed to obtain an accurate PC model and then the ones limiting the number of terms in the desired PC model.

Table 2. Number of terms $(\boldsymbol{M}+\mathbf{1})$ in a PC model (Equation (3)) with respect to the expansion order $\boldsymbol{P}$ and number of random parameters $N$ computed via Equation (15).

\begin{tabular}{cccccccc}
\hline$\downarrow \mathbf{N}$ & $\mathbf{P = 1}$ & $\mathbf{P = \mathbf { 2 }}$ & $\mathbf{P = 3}$ & $\mathbf{P}=\mathbf{4}$ & $\mathbf{P}=\mathbf{5}$ & $\mathbf{P}=\mathbf{6}$ & $\mathbf{P}=\mathbf{7}$ \\
\hline 1 & 2 & 3 & 4 & 5 & 6 & 7 & 8 \\
2 & 3 & 6 & 10 & 15 & 21 & 28 & 36 \\
3 & 4 & 10 & 20 & 35 & 56 & 84 & 120 \\
8 & 9 & 45 & 165 & 495 & 1287 & 3003 & 6435 \\
12 & 13 & 915 & 455 & 1820 & 6188 & 18,564 & 50,388 \\
\hline
\end{tabular}

\subsection{Efficient Sampling Strategies for High-Dimensional Problems}

Adopting sparse grids is an efficient method to reduce the number of samples $K$ needed to compute a PC model, as discussed in Section 4 for the spectral projection or collocation approach. Sparse grids based on the Smolyak algorithm [22,60,63] selects smaller subsets (or low order 
interactions) of the corresponding full tensor grids, based on suitable criteria. However, strategies based on sparse grids also have some drawbacks. First, little flexibility is available to choose the number and location in the stochastic space of the collocation nodes (predefined set of nodes). Furthermore, while sparse grids offer a comparable accuracy for high dimensions, they also suffer from the curse of dimensionality (though to a lesser extent). In particular, grids based on the Smolyak algorithm scales as $\left(2^{N}\right)^{P} / P$ ! [22], while isotropic sparse grids scale as $P^{\log N}$ [23] for comparable accuracy. More details about tensor products and sparse grids based on the Smolyak algorithm are given in Appendix A. Various adaptive sparse grid methods have been proposed in the literature to further mitigate the curse of dimensionality, such as nested sparse grids [62], weighted Smolyak algorithms [63], hierarchical [64] and data-driven [65] sparse grids, and the dimension adaptive approach [94].

Recently, there has been a growing interest in the research community about tensor-based methods [95] to solve high-dimensional UQ problems. Indeed, the application of such techniques has grown rapidly: micromagnetics [96-98], model order reduction [99,100], big data [101-103], signal processing [104-107], control design [108,109], and electronic design automation [93].

Basically, a tensor is a multi-dimensional (also known as multi-mode or multi-way) data array. In general, a d-th order tensor $\mathcal{A}$ of size $n_{1} \times n_{2} \times \cdots \times n_{d}$ can be denoted as $\mathcal{A} \in \mathbb{R}^{n_{1} \times n_{2} \times \cdots \times n_{d}}$, where the order of a tensor is the number of dimensions, also known as ways or modes. A vector $\mathbf{a} \in \mathbb{R}^{n}$ and a matrix $\mathbf{A} \in \mathbb{R}^{n_{1} \times n_{2}}$ can be considered as special cases of tensors of order one and two, respectively. The main benefit of tensor models is their multilinear structure giving a concise representation of the underlying structure of the corresponding tensor, which is often low-rank. Indeed, in general, the number of terms in a tensor grows exponentially with the dimension considered (the curse of dimensionality), which makes it difficult to explicitly store a tensor. Therefore, it becomes necessary to approximate higher-order tensors by suitable compressing schemes. This can be achieved by tensor decomposition (primarily low-rank), which is able to compress and represent a high-dimensional tensor by a smaller number of factors, significantly reducing the cost of storing high-dimensional data arrays: typically, $\boldsymbol{n}^{d}$ scalars are required to represent a tensor $\mathcal{A}$, whereas, if its low-rank decomposition exists, only $n d r$ scalars are required to store the same tensor-based on canonical decomposition, where $r$ is the tensor rank [103].

Various tensor decomposition techniques have been presented in the last several years $[95,104]$. A literature study of low-rank tensor approximation techniques is carried out in [110]. The most popular low-rank tensor decomposition techniques are the canonical polyadic, Tucker and tensor-train decompositions. The properties, applications, and challenges of these techniques are discussed in [93].

Note that, often, the major part of the variation in the data considered is governed by a small number of latent factors. In this case, it is possible to estimate the corresponding tensor efficiently (using only a small number of simulations) by exploiting its low-rank part or other properties (such as sparsity or symmetry [111]). This approach is referred to as tensor recovery, and several methods have been proposed in the literature. For example, the low-rank property and the sparsity of the PC expansion were utilized in [103] to estimate a full tensor by using a small number of simulations for the high-dimensional UQ of ICs and micro-electromechanical systems. In [111], an underlying sparse structure is exploited to recover a tensor by minimizing a suitable sparsity-measure. A canonical polyadic decomposition-based method for down-link channel estimation was proposed in [112] for millimeter wave MIMO-OFDM systems. A high-dimensional hierarchical UQ-based on ANOVA and tensor-train decomposition has been developed in [113].

\subsection{Optimization of PC Models}

As discussed before, in the PC framework, the number of terms grows exponentially with respect to the number of random variables, making it impossible to use standard linear truncation schemes in a high-dimensional parameter space. However, it is possible to reduce the number of terms in Equation (3) by using different approaches that rely on one principle: usually, not all the $M+1$ coefficients in a PC model are relevant in accurately determining stochastic variations. 
As discussed in Section 3.2, the PC basis functions can be computed as a product combination of orthogonal polynomials corresponding to each individual random variable: hence, basis functions given by the products of polynomials corresponding to different random variables describe the interactions among such variables. A hyperbolic truncation scheme is presented in [114], which considers the predominant interactions among low-degree polynomial PC basis functions. Hence, among all the $M+1$ coefficients in a PC model, only a suitable subset is considered and the rest of the coefficients are discarded, resulting in a sparse PC expansion. Such a hyperbolic truncation scheme selects the basis functions according to the following criteria:

$$
\|d\|_{q} \leq P \text { and }\|d\|_{q} \equiv\left(\sum_{k=1}^{N} i_{k}^{q}\right)^{1 / q}
$$

where $\boldsymbol{i}_{\boldsymbol{k}}$ is the index defined in (7) and $\mathbf{0}<\boldsymbol{q} \leq \mathbf{1}$. The parameter $\boldsymbol{q}$ defines the sparsity level: for values of $\boldsymbol{q}$ close to zero, the corresponding PC model retains only the low-order interactions, while a value of $\boldsymbol{q}=\mathbf{1}$ results in a PC model chosen according to (15). Note that choosing $\boldsymbol{q}=\mathbf{0}$ means keeping only the univariate polynomials among the entire basis functions (the ones depending only on one random variable at the time). The impact of $\boldsymbol{q}$ on the sparsity of the resulting PC model is illustrated in Figure 3, considering $\boldsymbol{N}=\mathbf{2}$ and $\boldsymbol{P}=5$.

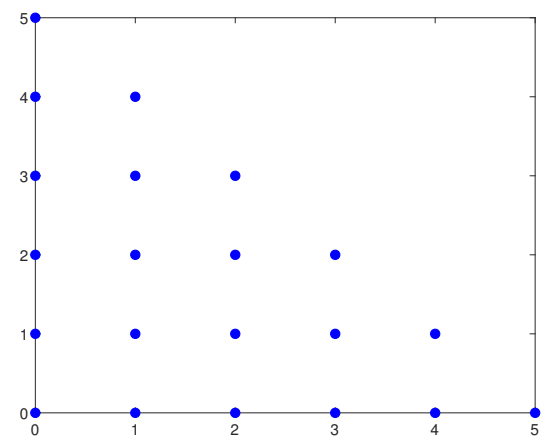

(a) $q=1, N=2, P=5$, set $=21$

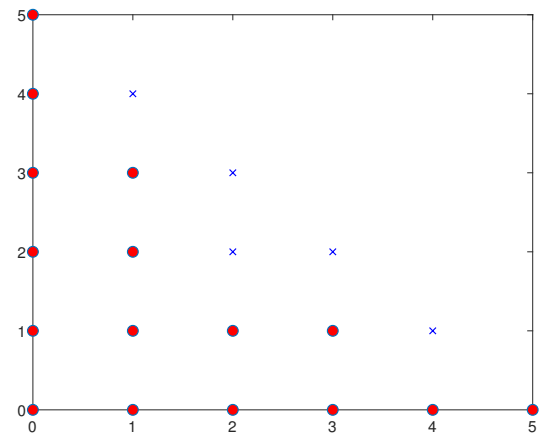

(b) $\boldsymbol{q}=0.75, N=2, P=5$, set $=16$

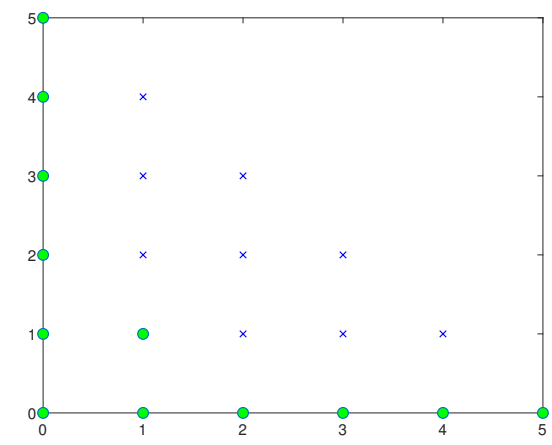

(c) $q=0.5, N=2, P=5$, set $=12$

Figure 3. The hyperbolic index set for different values of $\boldsymbol{q}$. The degree of polynomials for the two random variables is indicated in the $x$-and $y$-axes, while the blue exes $(\times)$ represent the corresponding polynomial. The circles indicate which polynomials are retained for a specific value of $\boldsymbol{q}$. Note that, if $q=0$, only the polynomials laying on the two axes are chosen. (a) shows polynomials retained for $q=1$; (b) shows polynomials retained for $\boldsymbol{q}=0.75$; (c) shows polynomials retained for $\boldsymbol{q}=0.5$. 
Moreover, in [115], an efficient adaptive algorithm is proposed to select a sparse basis by optimizing the hyperbolic truncation scheme, which has been applied to UQ of nonlinear circuits. To this purpose, a greedy iterative approach is used to adaptively increase $q$ until the corresponding PC model achieves the desired accuracy. Furthermore, the authors in [116] used the hyperbolic truncation scheme in a power flow application. This is used in combination with a least squares problem and by adding a penalty term, which further minimizes the high degree of interactions in an adaptive way.

Note that, sparse PC models can also be determined by using iterative algorithms not based on the hyperbolic truncation scheme. Such algorithms are very useful when the system can be approximated by a small number of terms (main interactions) that are not known upfront. The least angle regression (LAR) algorithm proposed in [40] iteratively determines the significant basis sets, improving the overall accuracy of the PC model. A weighted $\boldsymbol{\ell}_{\mathbf{1}}$-minimization approach was proposed in [41] to obtain sparse PC expansions suitable to solve differential equations with high-dimensional random inputs.

\section{Conclusions}

Thanks to its ability to accurately and efficiently capture stochastic variations, the PC expansion has been extensively studied and applied for UQ problems in the electrical and electronics domains. In this work, we provide an overview of the characteristics of PC-based methods and describe the unique features of the application of the PC expansion for different relevant UQ applications. In particular, the PC expansion is able to successfully tackle UQ problems with a relatively limited number of random variables but suffers from the curse of dimensionality in high-dimensional parameter spaces. Indeed, the number of basis functions in a PC model grows exponentially with respect to the number of random variables, by construction, affecting both the model complexity and computational cost of any PC-based methods. To tackle this issue, the research community is investigating several alternative approaches, such as adaptive sparse grids and low-rank tensor-based methods.

Author Contributions: A.K., D.S. and T.D. contributed to the design and implementation of the research. A.K. wrote the paper and acted as corresponding author.

Conflicts of Interest: The authors declare no conflict of interest.

\section{Appendix A. Numerical Quadrature}

Numerical quadrature techniques are widely used to approximate integrals in stochastic spectral methods. In this framework, a one-dimensional integral can be approximated by using a $k$-point quadrature formula as

$$
\int_{\Omega} \mathrm{f}(x) W(x) \mathrm{d} x \approx \sum_{i=1}^{k} \mathrm{f}\left(x_{i}\right) w_{i}
$$

where $\mathrm{f}(x)$ is a function, $W(x)$ is a weight function, $k$ is the total number of quadrature points chosen via suitable quadrature rules, and $w_{i}$ are the corresponding weights. The choice of the quadrature rules and weights depends on the type of weight function $W(x)$ : for the stochastic problems considered in this paper, $W(x)$ corresponds to a probability density function of a random variable $x$ on a set $\Omega \subseteq \mathbb{R}^{N=1}$, where $N$ is the input dimension. Examples of possible quadrature rules are the Gauss [117] and Clenshaw-Curtis [118] quadrature. Note that the Clenshaw-Curtis rule results in a nested set of quadrature points: for instance, given a quadrature rule $Q_{k}$, the construction of $Q_{2 k-1}$ requires only the computation of $k-\mathbf{1}$ additional points, as the values $Q_{k}$ can be reused. The weight $w_{i}$ for $i=1, \ldots, k$ is given by the $i$-th roots of the orthogonal polynomials of the weighting function. Hence, in order to solve (A1), it is sufficient to evaluate the function $\mathrm{f}(x)$ only on the quadrature nodes $x_{i}$ for $i=1, \ldots, K$.

Now, multi-dimensional integrals can be computed as

$$
\int_{\Omega} \mathrm{f}(\vec{x}) W(\vec{x}) \mathrm{d} \vec{x} \approx \sum_{i=1}^{K} \mathrm{f}\left(\vec{x}_{i}\right) w_{i}
$$


where $\Omega \subseteq \mathbb{R}^{N}$ for $N>\mathbf{1}$, and $K$ is the total number of quadrature points.

The $\boldsymbol{K}$ points and weights in a multi-dimensional case can be easily obtained by using a tensor product of the quadrature points and weights chosen for each variable $x \in \vec{x}$. However, the efficiency of the tensor product-based approach decreases rapidly with respect to $N$, since the function $f(\vec{x})$ must be evaluated for a large set of quadrature points.

Sparse grids based on the Smolyak algorithm $[60,61]$ are often employed to lessen the impact of the number of dimensions on the efficiency of numerical quadrature techniques.

Indeed, the Smolyak algorithm selects a subset of quadrature points from a full tensor product grid and the corresponding weights are computed as given in [60,61]. By using Smolyak sparse grids, the quadrature points are given by

$$
\mathbf{H}_{L, N}=\bigcup_{L-N+1 \leq|\vec{k}| \leq L} \Theta_{1}^{k_{1}} \times \cdots \times \Theta_{N}^{k_{N}}
$$

where the vector $\vec{k}$ is formed by the desired level (or order) used for each variable, here $|\vec{k}|=k_{1}+\cdots+k_{N}$, and $L$ is the maximum level assumed for the sparse grid. The symbol $\Theta$ denotes the set of quadrature points used in each dimension. Moreover, the Smolyak grid is nested if it is based on one-dimensional node schemes generating a nested set of points, e.g., the Clenshaw-Curtis quadrature. However, if non-nested quadrature rules are chosen for some of the parameters, the resulting sparse grid is not nested.

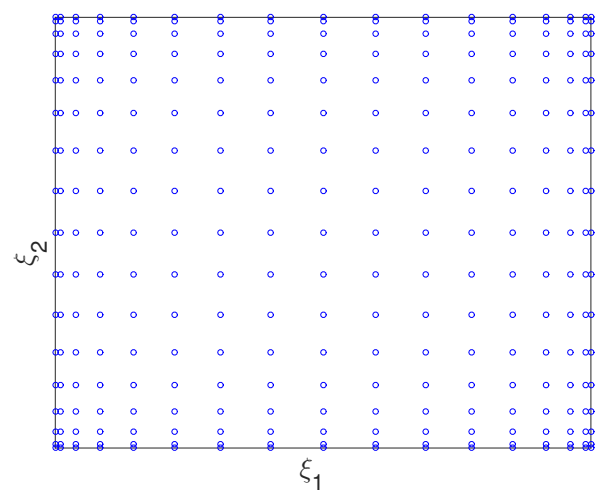

(a) Full tensor product.

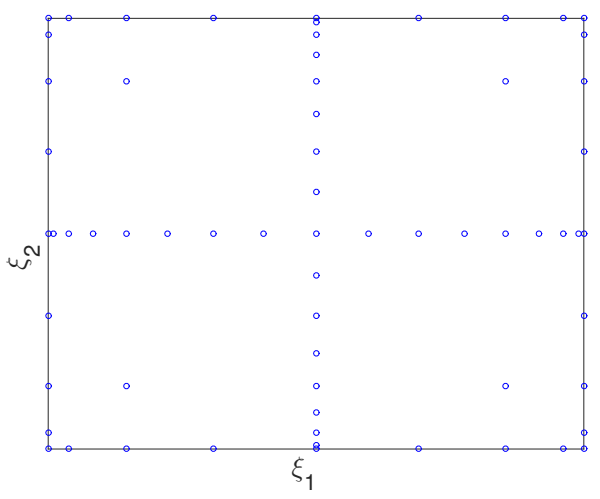

(b) Sparse grids on Clenshaw-Curtis.

Figure A1. (a) Two-dimensional tensor product based on 17 samples for each variable chosen via the Clenshaw-Curtis quadrature rule. (b) Corresponding Smolyak sparse grid $\mathbf{H}_{\mathbf{4}, \mathbf{2}}$.

In order to illustrate the grid construction based on the tensor product and sparse grid, a two-dimensional example is used here. For each variable, 17 points are chosen via the Clenshaw-Curtis quadrature rule (the resulting points are the extrema of the Chebyshev polynomials). The corresponding tensor product grid is shown in Figure A1a and is formed by $\mathbf{1 7} \times \mathbf{1 7}=\mathbf{2 8 9}$ points. The quadrature nodes for a level 4 sparse grid $\left(\mathbf{H}_{4,2}\right)$ are given by Equation (A3): only the 65 points in Figure A1b out of the total 289 are used.

\section{References}

1. Moore, G.E. Cramming more components onto integrated circuits. Electronics 1965, 38, 114-117.

2. Dhia, S.B.; Ramdani, M.; Sicard, E. Electromagnetic Compatibility of Integrated Circuits: Techniques for Low Emission and Susceptibility; Springer: New York, NJ, USA, 2005.

3. Maly, W. Cost of Silicon Viewed from VLSI Design Perspective. In Proceedings of the 31st Design Automation Conference, San Diego, CA, USA, 6-10 June 1994. 
4. Iwai, H.; Kakushima, K.; Wong, H. Challenges for Future Semiconductor Manufacturing. Int. J. High Speed Electron. Syst. 2006, 16, 43-81.

5. Nassif, S.R. Modeling and analysis of manufacturing variations. In Proceedings of the IEEE 2001 Custom Integrated Circuits Conference, San Diego, CA, USA, 9 May 2001; pp. 223-228.

6. Boning, D.S.; Balakrishnan, K.; Cai, H.; Drego, N.; Farahanchi, A.; Gettings, K.M.; Lim, D.; Somani, A.; Taylor, H.; Truque, D.; et al. Variation. IEEE Trans. Semicond. Manuf. 2008, 21, 63-71.

7. Tega, N.; Miki, H.; Pagette, F.; Frank, D.J.; Ray, A.; Rooks, M.J.; Haensch, W.; Torii, K. Increasing threshold voltage variation due to random telegraph noise in FETs as gate lengths scale to $20 \mathrm{~nm}$. In Proceedings of the Symposium on VLSI Technology, Honolulu, HI, USA, 16-18 June 2009; pp. 50-51.

8. Gong, F.; Liu, X.; Yu, H.; Tan, S.X.D.; Ren, J.; He, L. A Fast Non-Monte-Carlo Yield Analysis and Optimization by Stochastic Orthogonal Polynomials. ACM Trans. Des. Autom. Electron. Syst. 2012, 17, 10, doi:10.1145/2071356.2071366.

9. Bai, E.W. Adaptive quantification of model uncertainties by rational approximation. IEEE Trans. Autom. Control 1991, 36, 441-453.

10. De Vries, D.K.; Van Hof, P.M.J.D. Quantification of model uncertainty from data. Int. J. Robust Nonlinear Control 1994, 4, 301-319.

11. Hakvoort, R.G.; den Hof, M.J.V. Identification of probabilistic system uncertainty regions by explicit evaluation of bias and variance errors. IEEE Trans. Autom. Control 1997, 42, 1516-1528.

12. George, F. Monte Carlo: Concepts, Algorithms, and Applications; Springer: New York, NJ, USA, 1996.

13. Xiu, D.; Karniadakis, G.E. The Wiener-Askey Polynomial Chaos for Stochastic Differential Equations. SIAM J. Sci. Comput. 2002, 24, 619-644.

14. Blatman, G.; Sudret, B. An adaptive algorithm to build up sparse polynomial chaos expansions for stochastic finite element analysis. Probab. Eng. Mech. 2010, 25, 183-197.

15. Tao, J.; Zeng, X.; Cai, W.; Su, Y.; Zhou, D.; Chiang, C. Stochastic Sparse-grid Collocation Algorithm (SSCA) for Periodic Steady-State Analysis of Nonlinear System with Process Variations. In Proceedings of the Asia and South Pacific Design Automation Conference (ASPDAC), Yokohama, Japan, 23-26 January 2007; pp. 474-479.

16. Silly-Carette, J.; Lautru, D.; Wong, M.F.; Gati, A.; Wiart, J.; Hanna, V.F. Variability on the Propagation of a Plane Wave Using Stochastic Collocation Methods in a Bio Electromagnetic Application. IEEE Microw. Wirel. Compon. Lett. 2009, 19, 185-187.

17. Grigoriu, M. Reduced order models for random functions. Application to stochastic problems. Appl. Math. Model. 2009, 33, 161-175.

18. Fei, Z.; Huang, Y.; Zhou, J.; Xu, Q. Uncertainty Quantification of Crosstalk Using Stochastic Reduced Order Models. IEEE Trans. Electromagn. Compat. 2017, 59, 228-239.

19. Wiener, N. The Homogeneous Chaos. Am. J. Math. 1938, 60, 897-936.

20. Ghanem, R.G.; Spanos, P. Stochastic Finite Elements: A Spectral Approach; Springer: New York, NJ, USA, 1991.

21. Soize, C.; Ghanem, R. Physical Systems with Random Uncertainties: Chaos Representations with Arbitrary Probability Measure. SIAM J. Sci. Comput. 2004, 26, 395-410.

22. Xiu, D.; Hesthaven, J. High-Order Collocation Methods for Differential Equations with Random Inputs. SIAM J. Sci. Comput. 2005, 27, 1118-1139.

23. Eldred, M.S. Recent Advances in Non-Intrusive Polynomial Chaos and Stochastic Collocation Methods for Uncertainty Analysis and Design. In Proceedings of the 50th AIAA/ASME/ASCE/AHS/ASC Structures, Structural Dynamics, and Materials Conference, Palm Springs, CA, USA, 4-7 May 2009.

24. Xiu, D. Numerical Methods for Stochastic Computations: A Spectral Method Approach; Princeton University Press: Princeton, NJ, USA, 2010.

25. Web of Science. Available online: http:/ / webofknowledge.com/ (accessed on 15 November 2017).

26. Maricau, E.; Gielen, G. Analog IC Reliability in Nanometer CMOS (Analog Circuits and Signal Processing); Springer: New York, NJ, USA, 2013.

27. Onabajo, M.; Silva-Martinez, J. Analog Circuit Design for Process Variation-Resilient Systems-on-a-Chip; Springer: New York, NJ, USA, 2012.

28. Ochoa, J.; Cangellaris, A. Macro-modeling of electromagnetic domains exhibiting geometric and material uncertainty. Appl. Comput. Electromagn. Soc. J. 2012, 27, 80-87.

29. Sudret, B. Global sensitivity analysis using polynomial chaos expansion. Reliab. Eng. Syst. Saf. 2008, 93, 964-979. 
30. Sudret, B.; Mai, C. Computing derivative-based global sensitivity measures using polynomial chaos expansions. Reliab. Eng. Syst. Saf. 2015, 134, 241-250.

31. Petrocchi, A.; Kaintura, A.; Avolio, G.; Spina, D.; Dhaene, T.; Raffo, A.; Schreurs, D.M.M.P. Measurement Uncertainty Propagation in Transistor Model Parameters via Polynomial Chaos Expansion. IEEE Microw. Wirel. Compon. Lett. 2017, 27, 572-574.

32. Witteveen, J.A.S.; Bijl, H. Modeling Arbitrary Uncertainties Using Gram-Schmidt Polynomial Chaos. In Proceedings of the 44th AIAA Aerospace Sciences Meeting and Exhibit, Number AIAA-2006-0896, Reno, NV, USA, 9-12 January 2006.

33. Sudret, B. Polynomial chaos expansions and stochastic finite element methods. In Risk and Reliability in Geotechnical Engineering; Kok-Kwang Phoon, J.C., Ed.; CRC Press: Boca Raton, FL, USA, 2015.

34. Wan, X.; Karniadakis, G.E. Beyond Wiener-Askey Expansions: Handling Arbitrary PDFs. J. Sci. Comput. 2006, 27, 455-464.

35. Oladyshkin, S.; Nowak, W. Data-driven uncertainty quantification using the arbitraeq:mury polynomial chaos expansion. Reliab. Eng. Syst. Saf. 2012, 106, 179-190.

36. Loeve, M. Probability Theory I; Springer: New York, NJ, USA, 1977.

37. Spina, D.; Ferranti, F.; Dhaene, T.; Knockaert, L.; Antonini, G.; Vande Ginste, D. Variability Analysis of Multiport Systems Via Polynomial-Chaos Expansion. IEEE Trans. Microw. Theory Tech. 2012, 60, 2329-2338.

38. Zhang, Z.; El-Moselhy, T.A.; Elfadel, I.M.; Daniel, L. Stochastic Testing Method for Transistor-Level Uncertainty Quantification Based on Generalized Polynomial Chaos. IEEE Trans. Comput.-Aided Des. Integr. Circuits Syst. 2013, 32, 1533-1545.

39. Spina, D.; Ferranti, F.; Dhaene, T.; Knockaert, L.; Antonini, G. Polynomial chaos-based macromodeling of multiport systems using an input-output approach. Int. J. Numer. Model. Electron. Netw. Devices Fields 2015, 28, 562-581.

40. Blatman, G.; Sudret, B. Adaptive sparse polynomial chaos expansion based on least angle regression. J. Comput. Phys. 2011, 230, 2345-2367.

41. Peng, J.; Hampton, J.; Doostan, A. A weighted $\ell_{\mathbf{1}}$-minimization approach for sparse polynomial chaos expansions. J. Comput. Phys. 2014, 267, 92-111.

42. Strunz, K.; Su, Q. Stochastic Formulation of SPICE-type Electronic Circuit Simulation with Polynomial Chaos. ACM Trans. Model. Comput. Simul. 2008, 18, 15, doi:10.1145/1391978.1391981.

43. Su, Q.; Strunz, K. Stochastic Polynomial-Chaos-Based Average Modeling of Power Electronic Systems. IEEE Trans. Power Electron. 2011, 26, 1167-1171.

44. Pulch, R. Polynomial Chaos for Linear Differential Algebraic Equations with Random Parameters. Int. J. Uncertain. Quantif. 2011, 1, 223-240.

45. Stievano, I.S.; Manfredi, P.; Canavero, F.G. Parameters Variability Effects on Multiconductor Interconnects via Hermite Polynomial Chaos. IEEE Trans. Compon. Packag. Manuf. Technol. 2011, 1, 1234-1239.

46. Stievano, I.S.; Manfredi, P.; Canavero, F.G. Stochastic Analysis of Multiconductor Cables and Interconnects. IEEE Trans. Electromagn. Compat. 2011, 53, 501-507.

47. Vande Ginste, D.; De Zutter, D.; Deschrijver, D.; Dhaene, T.; Manfredi, P.; Canavero, F. Stochastic Modeling-Based Variability Analysis of On-Chip Interconnects. IEEE Trans. Compon. Packag. Manuf. Technol. 2012, 2, 1182-1192.

48. Spina, D.; Dhaene, T.; Knockaert, L.; Antonini, G. Polynomial Chaos-Based Macromodeling of General Linear Multiport Systems for Time-Domain Analysis. IEEE Trans. Microw. Theory Tech. 2017, 65, 1422-1433.

49. Lucor, D.; Su, C.H.; Karniadakis, G.E. Generalized polynomial chaos and random oscillators. Int. J. Numer. Methods Eng. 2004, 60, 571-596.

50. Monti, A.; Ponci, F.; Lovett, T. A polynomial chaos theory approach to the control design of a power converter. In Proceedings of the IEEE 35th Annual Power Electronics Specialists Conference, Aachen, Germany, 20-25 June 2004; Volume 6, pp. 4809-4813.

51. Pulch, R. Modelling and simulation of autonomous oscillators with random parameters. Math. Comput. Simul. 2011, 81, 1128-1143.

52. Rufuie, M.R.; Gad, E.; Nakhla, M.; Achar, R. Generalized Hermite Polynomial Chaos for Variability Analysis of Macromodels Embeddedin Nonlinear Circuits. IEEE Trans. Compon. Packag. Manuf. Technol. 2014, 4, 673-684. 
53. Spina, D.; De Jonghe, D.; Deschrijver, D.; Gielen, G.; Knockaert, L.; Dhaene, T. Stochastic Macromodeling of Nonlinear Systems Via Polynomial Chaos Expansion and Transfer Function Trajectories. IEEE Trans. Microw. Theory Tech. 2014, 62, 1454-1460.

54. Cheng, H.; Sandu, A. Collocation Least-squares Polynomial Chaos Method. In Proceedings of the 2010 Spring Simulation Multiconference, Orlando, FL, USA, 11-15 April 2010; pp. 1-6.

55. Berveiller, M.; Sudret, B.; Lemaire, M. Stochastic finite element: A non intrusive approach by regression. Eur. J. Comput. Mech. 2006, 15, 81-92.

56. Ghanem, R.; Spanos, P. A stochastic Galerkin expansion for nonlinear random vibration analysis. Probab. Eng. Mech. 1993, 8, 255-264.

57. Augustin, F.; Rentrop, P. Stochastic Galerkin techniques for random ordinary differential equations. Numer. Math. 2012, 122, 399-419.

58. Zhang, Z.; El-Moselhy, T.A.; Maffezzoni, P.; Elfadel, I.M.; Daniel, L. Efficient Uncertainty Quantification for the Periodic Steady State of Forced and Autonomous Circuits. IEEE Trans. Circuits Syst. II Express Briefs 2013, 60, 687-691.

59. Manfredi, P.; Canavero, F.G. Efficient Statistical Simulation of Microwave Devices Via Stochastic Testing-Based Circuit Equivalents of Nonlinear Components. IEEE Trans. Microw. Theory Tech. 2015, 63, 1502-1511.

60. Ganapathysubramanian, B.; Zabaras, N. Sparse Grid Collocation Schemes for Stochastic Natural Convection Problems. J. Comput. Phys. 2007, 225, 652-685.

61. Novak, E.; Ritter, K. Simple Cubature Formulas with High Polynomial Exactness. Constr. Approx. 1999, 15, 499-522.

62. Beddek, K.; Clenet, S.; Moreau, O.; Costan, V.; Menach, Y.L.; Benabou, A. Adaptive Method for Non-Intrusive Spectral Projection; Application on a Stochastic Eddy Current NDT Problem. IEEE Trans. Magn. 2012, $48,759-762$.

63. Agarwal, N.; Aluru, N. Weighted Smolyak algorithm for solution of stochastic differential equations on non-uniform probability measures. Int. J. Numer. Methods Eng. 2011, 85, 1365-1389.

64. Ma, X.; Zabaras, N. An adaptive hierarchical sparse grid collocation algorithm for the solution of stochastic differential equations. J. Comput. Phys. 2009, 228, 3084-3113.

65. Franzelin, F.; Pflüger, D. From Data to Uncertainty: An Efficient Integrated Data-Driven Sparse Grid Approach to Propagate Uncertainty. In Sparse Grids and Applications-Stuttgart 2014; Garcke, J., Pflüger, D., Eds.; Springer: Cham, Switzerland, 2016; pp. 29-49.

66. Maître, O.P.L.; Reagan, M.T.; Najm, H.N.; Ghanem, R.G.; Knio, O.M. A Stochastic Projection Method for Fluid Flow: II. Random Process. J. Comput. Phys. 2002, 181, 9-44.

67. Hosder, S.; Walters, R.; Balch, M. Efficient Sampling for Non-Intrusive Polynomial Chaos Applications with Multiple Uncertain Input Variables. In Proceedings of the 48th AIAA/ASME/ASCE/AHS/ASC Structures, Structural Dynamics, and Materials Conference, Honolulu, HI, USA, 23-26 April 2007.

68. Husslage, B.G.M.; Rennen, G.; Van Dam, E.R.; Den Hertog, D. Space-filling Latin hypercube designs for computer experiments. Optim. Eng. 2011, 12, 611-630.

69. Zein, S.; Colson, B.; Glineur, F. An Efficient Sampling Method for Regression-Based Polynomial Chaos Expansion. Commun. Comput. Phys. 2013, 13, 1173-1188.

70. Ahadi, M.; Roy, S. Sparse Linear Regression (SPLINER) Approach for Efficient Multidimensional Uncertainty Quantification of High-Speed Circuits. IEEE Trans. Comput.-Aided Des. Integr. Circuits Syst. 2016, 35, 1640-1652.

71. Prasad, A.K.; Ahadi, M.; Roy, S. Multidimensional Uncertainty Quantification of Microwave/RF Networks Using Linear Regression and Optimal Design of Experiments. IEEE Trans. Microw. Theory Tech. 2016, 64, 2433-2446.

72. Shin, Y.; Xiu, D. Nonadaptive Quasi-Optimal Points Selection for Least Squares Linear Regression. SIAM J. Sci. Comput. 2016, 38, A385-A411.

73. Hampton, J.; Doostan, A. Coherence Motivated Sampling and Convergence Analysis of Least-Squares Polynomial Chaos Regression. Comput. Methods Appl. Mech. Eng. 2015, 290, 73-97.

74. Manfredi, P.; Vande Ginste, D.; De Zutter, D.; Canavero, F.G. Uncertainty Assessment of Lossy and Dispersive Lines in SPICE-Type Environments. IEEE Trans. Compon. Packag. Manuf. Technol. 2013, 3, 1252-1258.

75. Zubac, Z.; Daniel, L.; De Zutter, D.; Vande Ginste, D. A Cholesky-Based SGM-MLFMM for Stochastic Full-Wave Problems Described by Correlated Random Variables. IEEE Antennas Wirel. Propag. Lett. 2017, 16, 776-779. 
76. Clénet, S. Approximation Methods to Solve Stochastic Problems in Computational Electromagnetics. In Proceedings of the Scientific Computing in Electrical Engineering (SCEE), Wuppertal, Germany, 22-25 July 2014; Springer: Cham, Switzerland, 2014.

77. Debusschere, B. Intrusive Polynomial Chaos Methods for Forward Uncertainty Propagation. In Handbook of Uncertainty Quantification; Ghanem, R., Higdon, D., Owhadi, H., Eds.; Springer: Cham, Switzerland, 2016.

78. Rossi, M.; Vande Ginste, D.; Rogier, H. Generalized polynomial chaos paradigms to model uncertainty in wireless links. In Proceedings of the 11th European Conference on Antennas and Propagation (EUCAP), Paris, France, 19-24 March 2017.

79. Wan, X.; Karniadakis, G.E. An adaptive multi-element generalized polynomial chaos method for stochastic differential equations. J. Comput. Phys. 2005, 209, 617-642.

80. Wan, X.; Karniadakis, G.E. Multi-Element Generalized Polynomial Chaos for Arbitrary Probability Measures. SIAM J. Sci. Comput. 2006, 28, 901-928.

81. Maître, O.L.; Najm, H.; Ghanem, R.; Knio, O. Multi-resolution analysis of Wiener-type uncertainty propagation schemes. J. Comput. Phys. 2004, 197, 502-531.

82. Maître, O.P.L.; Najm, H.N.; Pèbay, P.P.; Ghanem, R.G.; Knio, O.M. Multi-Resolution-Analysis Scheme for Uncertainty Quantification in Chemical Systems. SIAM J. Sci. Comput. 2007, 29, 864-889.

83. Spina, D.; Ferranti, F.; Antonini, G.; Dhaene, T.; Knockaert, L. Efficient Variability Analysis of Electromagnetic Systems Via Polynomial Chaos and Model Order Reduction. IEEE Trans. Compon. Packag. Manuf. Technol. 2014, 4, 1038-1051.

84. Yang, J.; Faverjon, B.; Peters, H.; Kessissoglou, N. Application of Polynomial Chaos Expansion and Model Order Reduction for Dynamic Analysis of Structures with Uncertainties. Procedia IUTAM 2015, 13, 63-70.

85. Sumant, P.; Wu, H.; Cangellaris, A.; Aluru, N. Reduced-Order Models of Finite Element Approximations of Electromagnetic Devices Exhibiting Statistical Variability. IEEE Trans. Antennas Propag. 2012, 60, 301-309.

86. Zhang, Z.; El-Moselhy, T.A.; Elfadel, I.M.; Daniel, L. Calculation of Generalized Polynomial-Chaos Basis Functions and Gauss Quadrature Rules in Hierarchical Uncertainty Quantification. IEEE Trans. Comput.-Aided Des. Integr. Circuits Syst. 2014, 33, 728-740.

87. Keiter, E.R.; Swiler, L.P.; Wilcox, I.Z. Gradient-Enhanced Polynomial Chaos Methods for Circuit Simulation. In Proceedings of the 11th International Conference on Scientific Computing in Electrical Engineering (SCEE), St. Wolfgang, Austria, 3-7 October 2016.

88. Ng, L.W.T.; Eldred, M. Multifidelity Uncertainty Quantification Using Non-Intrusive Polynomial Chaos and Stochastic Collocation. In Proceedings of the 53rd AIAA/ASME/ASCE/AHS/ASC Structures, Structural Dynamics and Materials Conference, Honolulu, HI, USA, 23-26 April 2012.

89. Palar, P.S.; Tsuchiya, T.; Parks, G.T. Multi-fidelity non-intrusive polynomial chaos based on regression. Comput. Methods Appl. Mech. Eng. 2016, 305, 579-606.

90. Manfredi, P.; Vande Ginste, D.; De Zutter, D.; Canavero, F.G. On the Passivity of Polynomial Chaos-Based Augmented Models for Stochastic Circuits. IEEE Trans. Circuits Syst. I Regul. Pap. 2013, 60, 2998-3007.

91. Ye, Y.; Spina, D.; Manfredi, P.; Vande Ginste, D.; Dhaene, T. A Comprehensive and Modular Stochastic Modeling Framework for the Variability-Aware Assessment of Signal Integrity in High-Speed Links. IEEE Trans. Electromagn. Compat. 2018, 60, 459-467.

92. Pham, T.A.; Gad, E.; Nakhla, M.S.; Achar, R. Decoupled Polynomial Chaos and Its Applications to Statistical Analysis of High-Speed Interconnects. IEEE Trans. Compon. Packag. Manuf. Technol. 2014, 4, 1634-1647.

93. Zhang, Z.; Batselier, K.; Liu, H.; Daniel, L.; Wong, N. Tensor Computation: A New Framework for High-Dimensional Problems in EDA. IEEE Trans. Comput.-Aided Des. Integr. Circuits Syst. 2017, 36, 521-536.

94. Tang, J.; Ni, F.; Ponci, F.; Monti, A. Dimension-Adaptive Sparse Grid Interpolation for Uncertainty Quantification in Modern Power Systems: Probabilistic Power Flow. IEEE Trans. Power Syst. 2016, 31, 907-919.

95. Kolda, T.G.; Bader, B.W. Tensor Decompositions and Applications. SIAM Rev. 2009, 51, 455-500.

96. Abert, C.; Exl, L.; Selke, G.; Drews, A.; Schrefl, T. Numerical methods for the stray-field calculation: A comparison of recently developed algorithms. J. Magn. Magn. Mater. 2013, 326, 176-185.

97. Exl, L.; Auzinger, W.; Bance, S.; Gusenbauer, M.; Reichel, F.; Schrefl, T. Fast stray field computation on tensor grids. J. Comput. Phys. 2012, 231, 2840-2850.

98. Falcó, A.; Hackbusch, W. On Minimal Subspaces in Tensor Representations. Found. Comput. Math. 2012, 12, 765-803. 
99. Nouy, A. Low-Rank Tensor Methods for Model Order Reduction. In Handbook of Uncertainty Quantification; Ghanem, R., Higdon, D., Owhadi, H., Eds.; Springer: Cham, Switzerland, 2016.

100. Liu, H.; Daniel, L.; Wong, N. Model Reduction and Simulation of Nonlinear Circuits via Tensor Decomposition. IEEE Trans. Comput.-Aided Des. Integr. Circuits Syst. 2015, 34, 1059-1069.

101. Vervliet, N.; Debals, O.; Sorber, L.; Lathauwer, L.D. Breaking the Curse of Dimensionality Using Decompositions of Incomplete Tensors: Tensor-based scientific computing in big data analysis. IEEE Signal Process. Mag. 2014, 31, 71-79.

102. Chen, D.; Hu, Y.; Wang, L.; Zomaya, A.Y.; Li, X. H-PARAFAC: Hierarchical Parallel Factor Analysis of Multidimensional Big Data. IEEE Trans. Parallel Distrib. Syst. 2017, 28, 1091-1104.

103. Zhang, Z.; Weng, T.W.; Daniel, L. Big-Data Tensor Recovery for High-Dimensional Uncertainty Quantification of Process Variations. IEEE Trans. Compon. Packag. Manuf. Technol. 2017, 7, 687-697.

104. Cichocki, A.; Mandic, D.; Lathauwer, L.D.; Zhou, G.; Zhao, Q.; Caiafa, C.; PHAN, H.A. Tensor Decompositions for Signal Processing Applications: From two-way to multiway component analysis. IEEE Signal Process. Mag. 2015, 32, 145-163.

105. Sidiropoulos, N.D.; Lathauwer, L.D.; Fu, X.; Huang, K.; Papalexakis, E.E.; Faloutsos, C. Tensor Decomposition for Signal Processing and Machine Learning. IEEE Trans. Signal Process. 2017, 65, 3551-3582.

106. Eeghem, F.V.; Sorensen, M.; Lathauwer, L.D. Tensor Decompositions With Several Block-Hankel Factors and Application in Blind System Identification. IEEE Trans. Signal Process. 2017, 65, 4090-4101.

107. Zhang, Z.; Aeron, S. Exact Tensor Completion Using t-SVD. IEEE Trans. Signal Process. 2017, 65, 1511-1526.

108. Stoev, J.; Ertveldt, J.; Oomen, T.; Schoukens, J. Tensor methods for MIMO decoupling and control design using frequency response functions. Mechatronics 2017, 45, 71-81.

109. Lu, J.; Li, H.; Liu, Y.; Li, F. Survey on semi-tensor product method with its applications in logical networks and other finite-valued systems. IET Control Theory Appl. 2017, 11, 2040-2047.

110. Grasedyck, L.; Kressner, D.; Tobler, C. A literature survey of low-rank tensor approximation techniques. GAMM-Mitt. 2013, 36, 53-78.

111. Gandy, S.; Recht, B.; Yamada, I. Tensor completion and low-n-rank tensor recovery via convex optimization. Inverse Probl. 2011, 27, 025010, doi:10.1088/0266-5611/27/2/025010.

112. Zhou, Z.; Fang, J.; Yang, L.; Li, H.; Chen, Z.; Blum, R.S. Low-Rank Tensor Decomposition-Aided Channel Estimation for Millimeter Wave MIMO-OFDM Systems. IEEE J. Sel. Areas Commun. 2017, 35, 1524-1538.

113. Zhang, Z.; Yang, X.; Oseledets, I.V.; Karniadakis, G.E.; Daniel, L. Enabling High-Dimensional Hierarchical Uncertainty Quantification by ANOVA and Tensor-Train Decomposition. IEEE Trans. Comput.-Aided Des. Integr. Circuits Syst. 2015, 34, 63-76.

114. Blatman, G. Adaptive Sparse Polynomial Chaos Expansions for Uncertainty Propagation and Sensitivity Analysis. Ph.D. Thesis, Université Blaise Pascal, Clermont-Ferrand, France, 2009.

115. Ahadi, M.; Prasad, A.K.; Roy, S. Hyperbolic polynomial chaos expansion (HPCE) and its application to statistical analysis of nonlinear circuits. In Proceedings of the IEEE 20th Workshop on Signal and Power Integrity (SPI), Turin, Italy, 8-11 May 2016; pp. 1-4.

116. Ni, F.; Nguyen, P.H.; Cobben, J.F.G. Basis-Adaptive Sparse Polynomial Chaos Expansion for Probabilistic Power Flow. IEEE Trans. Power Syst. 2017, 32, 694-704.

117. Golub, G.H.; Welsch, J.H. Calculation of Gauss quadrature rules. Math. Comput. 1969, 23, 221-230.

118. Clenshaw, C.W.; Curtis, A.R. A method for numerical integration on an automatic computer. Numer. Math. 1960, 2, 197-205.

(C) 2018 by the authors. Licensee MDPI, Basel, Switzerland. This article is an open access article distributed under the terms and conditions of the Creative Commons Attribution (CC BY) license (http:/ / creativecommons.org/licenses/by/4.0/). 